\title{
Local ensemble assimilation scheme with global constraints and conservation
}

\author{
Alexander Barth, Yajing Yan, Martin Canter, Aida Alvera-Azcárate, and Jean-Marie Beckers \\ University of Liege, AGO/GHER, Liege, Belgium (a.barth@ulg.ac.be)
}

Ensemble assimilation schemes applied in their original, global formulation have no problem in respecting linear conservation properties if the ensemble perturbations are setup accordingly. For realistic ocean systems, only a relatively small number of ensemble members can be calculated. A localization of the ensemble increment is thus necessary to filter out spurious long-range correlations. However, the conservation of the global property will be lost if the assimilation is performed locally since the conservation requires a coupling between model grid points, which is filtered out by the localization.

In the ocean, the distribution of observations is highly inhomogeneous. Systematic errors of the observed parts of the ocean state can lead to spurious systematic adjustments of the non-observed part of the ocean state due to data assimilation. As a result, global properties which should be conserved, increase or decrease in long-term simulations.

We propose an assimilation scheme (with stochastic or deterministic analysis steps) which is formulated globally (i.e. for the whole state vector) but where spurious long-range correlations can be filtered out. The scheme can thus be used to enforce global conservation properties and non-local observation operators. Both aspects are indeed linked since one can introduce the global conservation as a weak constraint by using a global observation operator. The conserved property becomes thus an observed value.

The proposed scheme is tested with the Kuramoto-Sivashinsky model which is conservative. The benefit compared to the traditional covariance localization scheme (with an ad-hoc step enforcing conservation) where observations are assimilated sequentially is shown. The assimilation scheme is suitable to be implemented on parallel computers where the number of available computing cores is a multiple of the ensemble size. 